\title{
Unfolded Protein Response in Lung Health and Disease
}

\author{
Nektarios Barabutis* \\ School of Basic Pharmaceutical and Toxicological Sciences, College of Pharmacy, University of Louisiana Monroe, Monroe, \\ LA, United States
}

The unfolded protein response (UPR) is a complex element, destined to protect the cells against a diverse variety of extracellular and intracellular challenges. UPR activation devises highly efficient responses to counteract cellular threats. If those activities fail, it will dictate cellular execution. The current work focuses on the role of UPR in pulmonary function, by immersing into the highly interrelated network that operates toward the endothelial barrier function. A highly sophisticated UPR manipulation shall reveal new therapeutic possibilities against inflammatory lung disease, such as acute lung injury and acute respiratory distress syndrome.

Keywords: endothelial dysfunction, ER stress, P53, inflammation, vasculature

\section{OPEN ACCESS}

Edited by:

Anne Hilgendorff,

Ludwig Maximilian University of

Munich, Germany

Reviewed by:

loannis Eleftherianos,

George Washington University,

United States

Izabela Zawlik,

University of Rzeszow, Poland

*Correspondence: Nektarios Barabutis

barabutis@ulm.edu

Specialty section:

This article was submitted to

Pulmonary Medicine,

a section of the journal

Frontiers in Medicine

Received: 07 April 2020

Accepted: 09 June 2020

Published: 30 July 2020

Citation:

Barabutis N (2020) Unfolded Protein

Response in Lung Health and

Disease. Front. Med. 7:344.

doi: 10.3389/fmed.2020.00344

\section{UNFOLDED PROTEIN RESPONSE}

The ribosomes of the endoplasmic reticulum (ER) produce secretory proteins, which are consequently subjected to modifications by cellular chaperones. The excessive abundance of misfolded proteins in the intracellular niche induces ER stress (1), which in turn induces the activation of the unfolded protein response (UPR) (2). This molecular machinery is in charge of protein synthesis, trafficking, and folding able to promote both cell survival and death (3). In the case of mild ER stress, UPR initiates survival responses (4). Upon severe circumstances, it will execute the cells by triggering lethal mechanisms (5-7).

Protein kinase RNA-like ER kinase (PERK), activating transcription factor 6 (ATF6), and the inositol-requiring enzyme-1 $\alpha(\operatorname{IRE} 1 \alpha)$ are the major components of the UPR $(8,9)$. The binding immunoglobulin protein (BiP) is a member of the heat shock protein (HSP) 70 , and it is destined to assist in the maturation and folding of nascent proteins (10). When those proteins bind to the $\mathrm{BiP}$, they are inactive. Indeed, the release of this protein will capacitate UPR (11).

Our genome encodes for IRE1 $\alpha$ and IRE1 $\beta$. IRE1 $\alpha$ is expressed ubiquitously, and it is essential for mice. IRE1 $\alpha$ knockout mice exhibit embryonic lethality. On the contrary, IRE1 $\beta$ knockout mice are viable (12-14). IRE1 associates with BiP when there is no evident ER stress. Upon situations of such stress, BiP will relocate from IRE1 to the misfolded ER protein and will initiate auto-phosphorylation and dimerization (15). Consequently, IRE1 $\alpha$ will cleave the X-box binding protein-1 (XBP1). This modification produces XBP1S, which is considered the active XBP1 form, strongly involved in ER membrane biogenesis, folding, and secretion (16).

PERK is structurally related to IRE1 $\alpha$ and exists as a monomer under unstressed conditions (17). In the ER, the excessive abundance of proteins which are not folded correctly activates PERK via dimerization and auto-phosphorylation (18). This modification will phosphorylate (I) the eukaryotic translation initiation factor 2-alpha (elf2a) and (II) the nuclear factor erythroid 2-related factor 2 (nrf2). All those events will activate ATF4 and nrf2 (19). PERK may also be activated by tau accumulation (20). 
ATF6 is a type 2 transmembrane transcription factor. ER stress initiates the release of BiP from ATF6 (9), which in turn will be cleaved by serine protease site-1, as well as by metalloprotease site-2. Those modifications will result in its activation (21). It has been reported that ATF6 isoforms are essential for life in certain organisms. Silencing of ATF $6 \alpha$ and ATF6 $\beta$ has been shown to be associated with lethal outcomes in nonhuman vertebrates. Moreover, genetic manipulations of the ATF6 cause crucial problems in the human eye, associated with dysfunctions in neuroretina and blindness (22).

UPR is considered a promising target for cardiovascular disease therapy. There are two different strategies to employ UPR toward the development of new drugs. The first method suggests to activate the adaptive pathway of UPR, so as to counteract ER stress. The second one indicates to suppress the proapoptotic components of UPR to promote cell survival (23). The current knowledge regarding the exact interrelations between the UPR components is incomplete, and it is speculated that such investigations may help utilize the UPR-mediated survival mechanisms to oppose cardiovascular disease $(16,24)$. In the following sections of our manuscript, we will refer to the role of UPR in both lung disease and health, in order to seed light to those events.

\section{UPR INDUCTION PROMOTES LUNG DISEASE}

In pulmonary fibrosis, IRE1a kinase/RNase suppression exerts anti-fibrotic effects in the lungs. The exposure of murine lungs to bleomycin caused ER stress, as reflected in the activation of IRE1a and the consequent development of pulmonary fibrosis. The small-molecule IRE1 $\alpha$ kinase-inhibiting RNase attenuator (KIRA) 7 and 8 prevented lung fibrosis; thus, those observations revealed that IRE $1 \alpha$ is probably a therapeutic target for lung fibrosis (25).

The activation of PERK has been associated with lung fibroblast differentiation due to endothelin 1 (ET-1) or thrombin. In WI-38 human cells, which are embryonic lung fibroblasts, ET-1 and thrombin induced the expression of the UPR markers PERK and BiP. Moreover, they augmented the levels of $\alpha$-smooth muscle actin ( $\alpha$-SMA) and collagen (I, IV) and activated the cJun N-terminal kinase (JNK). Those effects were diminished by PERK knockdown. Similar observations were observed in an in vivo study of bleomycin-induced pulmonary fibrosis (26).

Inhibition of the IRE1 $\alpha$ signaling pathway blocked ER expansion via the XBP-1-dependent pathway, as well as the activation of myofibroblasts by the tumor growth factor $\beta$. Moreover, it lessened the fibrosis in both the liver and the skin, reverted the fibrotic effects in myofibroblasts, and affected the suppressive effects of miR-150 in $\alpha$-SMA expression (27).

The lungs of patients with idiopathic pulmonary fibrosis are subjected to increased ER stress, and investigations aimed to examine whether ER stress causes lung fibrosis (28). The surfactant protein C (L188Q SFTPC), which appears in interstitial pneumonia, was employed in those studies. This mutated protein induced ER stress, since the expression of
$\mathrm{BiP}$ and XBP1 was increased. The intra-tracheal injection of bleomycin in the L188Q SFTPC-expressing mice resulted in lung fibrosis. Since those mice demonstrated increased apoptotic death of the alveolar epithelial cells, as well as fibrotic lungs, it was assumed that ER stress may cause lung fibrosis (11). Interestingly, the pulmonary UPR is activated due to a plethora of parameters with associated lung fibrosis. Among those factors are infections with viruses, as well as aging (29).

Idiopathic pulmonary fibrosis (IPF) is characterized by elevated levels of GRP78. Cells were exposed to TGF- $\beta 1$ to evaluate the effects of ER stress in the $\alpha$-smooth muscle actin and collagen type I expression in the fibroblasts. This growth factor elevated the expression of three different ER stress markers, namely, GRP78, XBP-1, and ATF6 $\alpha$. Moreover, it exerted similar effects in the expression of $\alpha$-SMA and collagen type I (30).

Chronic obstructive pulmonary disease (COPD) and chronic bronchitis are characterized by increased oxidative stress (31). Inflammatory processes, apoptotic phenomena, and autophagy are major factors in the devastations due to COPD (32). Although PERK induction has been strongly involved in the development of COPD, it appears that the involvement of IRE1 and ATF6 toward those events has not been delineated yet (33).

The lungs of individuals exposed to the smoke of cigarettes, as well as those of COPD patients, express elevated BiP levels. Thus, it was proposed that pulmonary BiP secretion may explain the increase in serum BiP of COPD patients. Researchers measured $\mathrm{BiP}$ levels in the bronchoalveolar lavage fluid (BALF) of chronic and nonsmokers. It was revealed that $\mathrm{BiP}$ was increased in the case of smokers and that the human airway epithelial cells of the smokers secreted $\mathrm{BiP}$ compared to healthy individuals. Thus, it was suggested that $\mathrm{BiP}$ expression in the lungs may serve as a marker of lung injury due to smoke (34).

Inhibition of ATF6 activation opposes the development of PAH. ATF6 activation causes upregulation of the neurite outgrowth inhibitor (Nogo), a protein responsible for ER maintenance. Nogo induction causes ER disruption to inhibit key calcium-sensitive enzymes involved in the progression of pulmonary hypertension ( $\mathrm{PH})$. Mice lacking Nogo appear to be phenotypically normal but are resistant to hypoxia-induced PAH (35).

Endothelial cell (EC) inflammation and barrier dysfunction are critical events in the pathogenesis of acute lung injury (ALI) and acute respiratory distress syndrome (36). Preconditioning of human pulmonary artery endothelial cells (HPAEC) to ER stress alleviated EC inflammation. BiP silencing inhibited NF-кB activation. Moreover, pre-exposure to SubAB resulted in lessened expression of several inflammatory mediators. Interestingly, $\mathrm{BiP}$ suppression resulted in the restoration of endothelial permeability. Those findings suggest the important role of $\mathrm{BiP}$ in the NF-кB-mediated endothelial inflammation (37).

A significant risk factor for ARDS development is obesity. It has been shown that in obese mice, there is significant endothelial dysfunction in the lungs. Those obese rodents exert an enhanced susceptibility to LPS-induced lung damage. Moreover, they present increased ER stress in their lung cells, similar to those changes due to exposure to tunicamycin. ER stress reduction protected those obese mice against LPS-induced ALI (38). 
Increased ER stress levels due to MAPK induction have been involved in the development of cystic fibrosis (CF). This atypical UPR activation was not associated with the PERK-eIF2 $\alpha$ induction. However, the CF cells exerted a hyper-inflammatory phenotype. Salubrinal, a selective elF2a inhibitor, weakened the inflammatory responses due to flagellin (immune activator) and Pseudomonas aeruginosa. Moreover, the abundance of IL-6 was dependent on the activation of the p38 MAPK pathway (39).

ER stress contributes to the development of bronchial asthma by regulating NF- $\kappa \mathrm{B}$ activation. The ER stress markers in peripheral blood mononuclear cell and BALF fluid from asthmatic patients were elevated compared to that of healthy individuals. In mice, the chemical chaperone 4-phenylbutyric acid, which alleviates ER stress, counteracted the translocation of NF- $\kappa$ ? Moreover, it reduced the levels of inflammatory cytokines and dendritic cell infiltration (40).

In malignant tissues, it is not certain whether UPR suppresses tumor growth or protects tumor cells by facilitating adaptive survival responses $(41,42)$. In cases of breast cancer, UPR activation appears to be protective and has been associated with anti-estrogen resistance. On the other hand, hyperactivation of the anticipatory UPR pathway in cancers converts it from cytoprotective to cytotoxic (23). In conclusion, the previously mentioned studies support the deteriorating role of UPR induction in the lungs and support the concept that effective inhibition of ER stress may serve as a promising therapeutic strategy toward lung disease.

\section{UPR INDUCTION PROMOTES LUNG HEALTH}

In the porcine reproductive and respiratory syndrome, IRE1 $\alpha$ activities due to ER stress were associated with TNF- $\alpha$ production. Indeed, PERK suppressed this growth factor and protected against heart failure and lung remodeling (43). PERK KO mice were used to test the effects of experimental induced lung fibrosis. TAC (transverse aortic constriction)induced congestive heart failure (CHF) caused an increase in FM small arteries in all groups. However, the number of those arteries was larger in the PERK KO mice than in the control mice. Furthermore, both wild-type and PERK KO mice exhibited a significant decrease in NM small arteries. However, the number of NM small arteries was lower in PERK KO. Thus, the TACinduced lung fibrosis was exacerbated in the lungs of PERK KO animals (44).

In knock-in mice that express a mutant $\mathrm{BiP}$, it was revealed that this protein plays a critical role in the surfactant of the lungs. The expression of this mutant $\mathrm{BiP}$ in newborns resulted in respiratory failure. Indeed, UPR failure to increase lung susceptibility to hypoxia and ischemia leads to neonatal respiratory failure, indicating that the UPR of alveolar cells is crucial for normal ER function and overload due to regular growth and development (45).

CHOP induction due to double-stranded RNA-activated protein kinase (PKR) suppresses lung injury due to hyperoxia. MLE-12 cells (lung epithelial cells) were exposed to hyperoxic conditions, which in turn elevated both $\mathrm{CHOP}$ expression and PKR activation. Interestingly, PKR suppression reduced the hyperoxia-induced CHOP expression. Moreover, hyperoxia induced both lung PKR phosphorylation and CHOP. Mice that did not express $\mathrm{CHOP}$ (CHOP KO mice) presented respiratory dysfunction due to lung edema and increased endothelial permeability. Thus, this study supported the protective role of CHOP in the hyperoxia-induced lung dysfunction (1).

Newborns with respiratory stress, when exposed to hyperoxic conditions, are susceptible to the development of lung injury and bronchopulmonary dysplasia. ERp57 is an ER thiol oxidoreductase, which is recruited to substrates through its association with calnexin and calreticulin. Both are molecular chaperones. Calnexin retains unfolded or unassembled N-linked glycoproteins in the ER. Calreticulin participates in various cellular processes, and it was first identified as a $\mathrm{Ca}^{2+}$-binding protein. Knockdown of ERp57 was associated with an increase in $\mathrm{BiP}$ expression levels and protects against apoptosis due to tunicamycin and hyperoxia (46).

Rhinovirus (RV) infection may result in CF. Studies detected an induction of $\mathrm{BiP}$ and $\mathrm{CHOP}$ in $\mathrm{CF}$ lungs after $\mathrm{RV}$ infection. UPR induction after treatment with pharmacologic UPR inducers prior to RV infection protected the cells against cell death. Hence, it was suggested that UPR induction may control respiratory virus replication (47).

DnaJ 4 (ERdj4) is a $\mathrm{BiP}$ cochaperone. It removes misfolded proteins from the ER lumen when the cells are exposed to increased levels of toxic factors. A mutated form of ERdj4, which is not functional, caused the death of mice. Those fatalities occurred because of hypoglycemia, associated with abnormal growth. The animals that did not die exerted levels of constitutive ER stress in several tissues, including the lungs. Those studies suggested the important role of BiP in lung survival (48).

GHRH antagonists are UPR inducers and have been recently shown to support endothelial barrier function by suppressing major inflammatory pathways (i.e., ERK1/2, JAK2/STAT3) (49) as well as by deactivating cofilin (50). The GHRH antagonist MIA-602 inhibited fibrosis and inflammation in mice subjected to bleomycin. All the animals inflicted with bleomycin were severely inflamed and presented respiratory abnormalities. Remarkably, the GHRH antagonist MIA-602 counteracted those effects both in vivo and in vitro and suppressed the abundance of major inflammatory markers. That antagonist has also prominently suppressed multiple inflammatory genes (51).

It was recently shown in lung cells that P53 opposes the LPS-inflicted lung endothelial barrier dysfunction, by mediating the RhoA/Rac signaling $(36,52,53)$. UPR modulation in lung cells affected P53 expression levels in a positive manner (54). UPR induction elevated the P53 expression levels, while UPR suppressors reduced them. Thus, it was speculated that P53 possibly protects the lung vasculature against ALI/ARDS, at least in part, by inducing UPR (55). Furthermore, the inhibition of Hsp90 has been shown to induce UPR (56). Those compounds induce P53, which in turn orchestrates robust anti-inflammatory responses (57-59).

Remarkably, UPR exerts a protective role in other tissues than lungs. Norartocarpin (NOR) induced the activity and stability of 
Nrf2, which has been shown to alleviate pathological outcomes of ER stress. Those effects ware associated with the manipulation of the following molecular pathways, which are crucial for cellular existence and survival: ERK1/2, phosphatidylinositol4,5-bisphosphate 3-kinase, protein kinase C, and PERK. The latter kinase, as previously mentioned, is an essential component of the UPR (60).

IRE1 has been shown to mediate the protective effects of LPS on myocardial ischemia-reperfusion injury. The LPS was administered in low doses. Rodents (rats) and cells were pretreated with a low dose of LPS prior to myocardial I/R injury. Unexpectedly, the administration of LPS at low doses did not harm the cells and did not significantly affect the animals. Indeed, it attenuated myocardial apoptosis. Proteins closely associated with IRE1 were increased during I/R injury. Such proteins are BiP, phospho-ASK1, and phospho-JNK. However, those effects were reduced by the LPS treatment (14).

The crucial role of $\mathrm{CHOP}$ against inflammation was reflected in the fact that CHOP deficiency in mice (CHOP KO) resulted in more significant kidney injury due to LPS, as compared to the wild-type animals. Those effects were associated with increased inflammation responses. Moreover, those LPS-inflicted animals contained a higher amount of renal neutrophil infiltrates compared to the wild-type counteracts. In the kidneys of those mice, increased NF- $\kappa \mathrm{B}$ activation and significant upregulation of pro-inflammatory genes were detected. Moreover, LPS treatment elevated the CHOP expression levels in wild-type mice (glomeruli, podocytes). Thus, the authors speculated that the increased CHOP in the kidneys of mice may be protective against AKI and oppose inflammation (61).

It was recently reported that the noncanonical mitochondrial UPR impairs placental oxidative phosphorylation in earlyonset preeclampsia. Thus, understanding mitochondrial stress may provide new insights regarding that pathology (62). The beneficial activities of GHRH antagonists in breast and prostate experimental models of disease have been associated with the induction of UPR and P53 $(50,55)$.

\section{CONCLUSIONS}

The role of UPR in the lungs is diverse. Based on the previously referenced literature, it is evident that UPR may protect the lungs

\section{REFERENCES}

1. Lozon TI, Eastman AJ, Matute-Bello G, Chen P, Hallstrand TS, Altemeier WA. PKR-dependent CHOP induction limits hyperoxia-induced lung injury. Am J Physiol Lung Cell Mol Physiol. (2011) 300:L422-9. doi: 10.1152/ajplung.00166.2010

2. Amen OM, Sarker SD, Ghildyal R, Arya A. Endoplasmic reticulum stress activates unfolded protein response signaling and mediates inflammation, obesity, and cardiac dysfunction: therapeutic and molecular approach. Front Pharmacol. (2019) 10:977. doi: 10.3389/fphar.2019.00977

3. Wang S, Kaufman RJ. The impact of the unfolded protein response on human disease. J Cell Biol. (2012) 197:857-67. doi: 10.1083/jcb.201110131

4. Barabutis N. Heat shock protein 90 inhibition in the inflamed lungs. Cell Stress Chaperones. (2020) 25:195-7. doi: 10.1007/s12192-020-01069-1 against human diseases or potentiate the intensity of various pathologies. In our opinion, the majority of the researchers are focused on the pathophysiological outcomes of UPR activation in lung microvasculature. Thus, most of the studies are targeted toward the suppression of UPR activation or alternatively aim to induce severe ER responses, which inevitably cause cell death.

In a similar fashion, P53 can kill the cells upon intense environmental and extracellular factors $(36,63)$, but its mild induction delivers protective effects against lung hyperpermeability (58). An increased production of reactive oxygen species may either promote lung pathologies or kill the cancer cells (64). Based on recent observations, we feel that the delineation of UPR signaling in the lungs may reveal new therapeutic possibilities toward lung disease and reveal new targets for lung disease, including ALI/ARDS (56, 65, 66). Experimental murine models of ALI/ARDS with endothelial specific mutations in control of the activation/deactivation of UPR branches shall be used to investigate the unexplored depths of the UPR universe and reveal potential therapies against respiratory dysfunctions.

\section{DATA AVAILABILITY STATEMENT}

The original contributions presented in the study are included in the article/supplementary materials, further inquiries can be directed to the corresponding author/s.

\section{AUTHOR CONTRIBUTIONS}

The author confirms being the sole contributor of this work and has approved it for publication.

\section{FUNDING}

NB research was supported by (I) R\&D, Research Competitiveness Subprogram (RCS), Louisiana Board of Regents through the Board of Regents Support Fund (LEQSF(2019-22)-RD-A-26), and (II) National Institute of General Medical Sciences of the National Institutes of Health (5 P20 GM103424-15 and 3 P20 GM103424-15S1). 
9. Glembotski CC, Rosarda JD, Wiseman RL. Proteostasis and beyond: ATF6 in Ischemic Disease. Trends Mol Med. (2019) 25:538-50. doi: 10.1016/j.molmed.2019.03.005

10. Ha TK, Hansen AH, Kildegaard HF, Lee GM. BiP inducer X: an ER stress inhibitor for enhancing recombinant antibody production in $\mathrm{CHO}$ cell culture. Biotechnol J. (2019) 14:e1900130. doi: 10.1002/biot.201900130

11. Lawson WE, Cheng DS, Degryse AL, Tanjore H, Polosukhin VV, Xu XC, et al. Endoplasmic reticulum stress enhances fibrotic remodeling in the lungs. Proc Natl Acad Sci USA. (2011) 108:10562-7. doi: 10.1073/pnas.1107559108

12. Chen L, Jarujaron S, Wu X, Sun L, Zha W, Liang G, et al. HIV protease inhibitor lopinavir-induced TNF-alpha and IL-6 expression is coupled to the unfolded protein response and ERK signaling pathways in macrophages. Biochem Pharmacol. (2009) 78:70-7. doi: 10.1016/j.bcp.2009.03.022

13. Chen Y, Brandizzi F. IRE1: ER stress sensor and cell fate executor. Trends Cell Biol. (2013) 23:547-55. doi: 10.1016/j.tcb.2013.06.005

14. $\mathrm{Wu} \mathrm{T}$, Jiang $\mathrm{N}$, Ji $\mathrm{Z}$, Shi G. The IRE1 signaling pathway is involved in the protective effect of low-dose LPS on myocardial ischemia-reperfusion injury. Life Sci. (2019) 231:116569. doi: 10.1016/j.lfs.2019.116569

15. Minamino T, Kitakaze M. ER stress in cardiovascular disease. J Mol Cell Cardiol. (2010) 48:1105-10. doi: 10.1016/j.yjmcc.2009.10.026

16. Minamino T, Komuro I, Kitakaze M. Endoplasmic reticulum stress as a therapeutic target in cardiovascular disease. Circ Res. (2010) 107:1071-82. doi: 10.1161/CIRCRESAHA.110.227819

17. Wang MG, Fan RF, Li WH, Zhang D, Yang DB, Wang ZY, et al. Activation of PERK-eIF2alpha-ATF4-CHOP axis triggered by excessive ER stress contributes to lead-induced nephrotoxicity. Biochim Biophys Acta Mol Cell Res. (2019) 1866:713-26. doi: 10.1016/j.bbamcr.2018.12.002

18. Pandey VK, Mathur A, Khan MF, Kakkar P. Activation of PERK-eIF2alphaATF4 pathway contributes to diabetic hepatotoxicity: Attenuation of ER stress by Morin. Cell Signal. (2019) 59:41-52. doi: 10.1016/j.cellsig.2019.03.008

19. Alasiri G, Jiramongkol Y, Zona S, Fan LY, Mahmud Z, Gong G, et al. Regulation of PERK expression by FOXO3: a vulnerability of drug-resistant cancer cells. Oncogene. (2019) 38:6382-98. doi: 10.1038/s41388-019-0890-7

20. Bruch J, Xu H, Rosler TW, De Andrade A, Kuhn PH, Lichtenthaler SF, et al. PERK activation mitigates tau pathology in vitro and in vivo. EMBO Mol Med. (2017) 9:371-84. doi: 10.15252/emmm.201606664

21. Fass D. Going for the Golgi: small PDI protein helps ATF6 perform better under stress. EMBO J. (2019) 38:e102743. doi: 10.15252/embj.2019102743

22. Kroeger H, Grimsey N, Paxman R, Chiang WC, Plate L, Jones Y, et al. The unfolded protein response regulator ATF6 promotes mesodermal differentiation. Sci Signal. (2018) 11:eaan5785. doi: 10.1126/scisignal.aan5785

23. Shapiro DJ, Livezey M, Yu L, Zheng X, Andruska N. Anticipatory UPR activation: a protective pathway and target in cancer. Trends Endocrinol Metab. (2016) 27:731-41. doi: 10.1016/j.tem.2016.06.002

24. Park SW, Ozcan U. Potential for therapeutic manipulation of the UPR in disease. Semin Immunopathol. (2013) 35:351-73. doi: $10.1007 / \mathrm{s} 00281-013-0370-\mathrm{Z}$

25. Thamsen M, Ghosh R, Auyeung VC, Brumwell A, Chapman HA, Backes BJ, et al. Small molecule inhibition of IRE1alpha kinase/RNase has anti-fibrotic effects in the lung. PLoS ONE. (2019) 14:e0209824. doi: 10.1371/journal.pone.0209824

26. Chen YC, Chen BC, Huang HM, Lin SH, Lin CH. Activation of PERK in ET-1and thrombin-induced pulmonary fibroblast differentiation: inhibitory effects of curcumin. J Cell Physiol. (2019). doi: 10.1002/jcp.28256. [Epub ahead of print].

27. Heindryckx F, Binet F, Ponticos M, Rombouts K, Lau J, Kreuger J, et al. Endoplasmic reticulum stress enhances fibrosis through IRE1alpha-mediated degradation of miR-150 and XBP-1 splicing. EMBO Mol Med. (2016) 8:72944. doi: 10.15252/emmm.201505925

28. Delbrel E, Soumare A, Naguez A, Label R, Bernard O, Bruhat A, et al. HIF-1alpha triggers ER stress and CHOP-mediated apoptosis in alveolar epithelial cells, a key event in pulmonary fibrosis. Sci Rep. (2018) 8:17939. doi: 10.1038/s41598-018-36063-2

29. Marciniak SJ. Endoplasmic reticulum stress in lung disease. Eur Respir Rev. (2017) 26:170018. doi: 10.1183/16000617.0018-2017

30. Baek HA, Kim DS, Park HS, Jang KY, Kang MJ, Lee DG, et al. Involvement of endoplasmic reticulum stress in myofibroblastic differentiation of lung fibroblasts. Am J Respir Cell Mol Biol. (2012) 46:731-9. doi: $10.1165 / \mathrm{rcmb} .2011-01210 \mathrm{O}$

31. Ribeiro CM, O'neal WK. Endoplasmic reticulum stress in chronic obstructive lung diseases. Curr Mol Med. (2012) 12:872-82. doi: 10.2174/156652412801318791

32. Yoshida T, Tuder RM. Pathobiology of cigarette smoke-induced chronic obstructive pulmonary disease. Physiol Rev. (2007) 87:1047-82. doi: 10.1152/physrev.00048.2006

33. Min T, Bodas M, Mazur S, Vij N. Critical role of proteostasis-imbalance in pathogenesis of COPD and severe emphysema. J Mol Med. (2011) 89:577-93. doi: 10.1007/s00109-011-0732-8

34. Aksoy MO, Kim V, Cornwell WD, Rogers TJ, Kosmider B, Bahmed K, et al. Secretion of the endoplasmic reticulum stress protein, GRP78, into the BALF is increased in cigarette smokers. Respir Res. (2017) 18:78. doi: 10.1186/s12931-017-0561-6

35. Dromparis P, Paulin R, Stenson TH, Haromy A, Sutendra G, Michelakis ED. Attenuating endoplasmic reticulum stress as a novel therapeutic strategy in pulmonary hypertension. Circulation. (2013) 127:115-25. doi: 10.1161/CIRCULATIONAHA.112.133413

36. Uddin MA, Barabutis N. P53: the endothelium defender. J Cell Biochem. (2019) 120:10952-5. doi: 10.1002/jcb.28511

37. Leonard A, Paton AW, El-Quadi M, Paton JC, Fazal F. Preconditioning with endoplasmic reticulum stress ameliorates endothelial cell inflammation. PLoS ONE. (2014) 9:e110949. doi: 10.1371/journal.pone.0110949

38. Shah D, Romero F, Guo Z, Sun J, Li J, Kallen CB, et al. Obesityinduced endoplasmic reticulum stress causes lung endothelial dysfunction and promotes acute lung injury. Am J Respir Cell Mol Biol. (2017) 57:204-15. doi: 10.1165/rcmb.2016-0310OC

39. Blohmke CJ, Mayer ML, Tang AC, Hirschfeld AF, Fjell CD, Sze MA, et al. Atypical activation of the unfolded protein response in cystic fibrosis airway cells contributes to p38 MAPK-mediated innate immune responses. J Immunol. (2012) 189:5467-75. doi: 10.4049/jimmunol.11 03661

40. Kim SR, Kim DI, Kang MR, Lee KS, Park SY, Jeong JS, et al. Endoplasmic reticulum stress influences bronchial asthma pathogenesis by modulating nuclear factor kappaB activation. J Allergy Clin Immunol. (2013) 132:1397408. doi: 10.1016/j.jaci.2013.08.041

41. Hetz C, Zhang K, Kaufman RJ. Mechanisms, regulation and functions of the unfolded protein response. Nat Rev Mol Cell Biol. (2020) 21:421-38. doi: 10.1038/s41580-020-0250-Z

42. Nonogaki H. The long-standing paradox of seed dormancy unfolded? Trends Plant Sci. (2019) 24:989-98. doi: 10.1016/j.tplants.2019.06.011

43. Chen WY, Schniztlein WM, Calzada-Nova G, Zuckermann FA. Genotype 2 strains of porcine reproductive and respiratory syndrome virus dysregulate alveolar macrophage cytokine production via the unfolded protein response. J Virol. (2018) 92:e01251-17. doi: 10.1128/JVI.01251-17

44. Liu X, Kwak D, Lu Z, Xu X, Fassett J, Wang H, et al. Endoplasmic reticulum stress sensor protein kinase R-like endoplasmic reticulum kinase (PERK) protects against pressure overload-induced heart failure and lung remodeling. Hypertension. (2014) 64:738-44. doi: 10.1161/HYPERTENSIONAHA.114.03811

45. Mimura N, Hamada H, Kashio M, Jin H, Toyama Y, Kimura K, et al. Aberrant quality control in the endoplasmic reticulum impairs the biosynthesis of pulmonary surfactant in mice expressing mutant BiP. Cell Death Differ. (2007) 14:1475-85. doi: 10.1038/sj.cdd.4402151

46. Xu D, Perez RE, Rezaiekhaligh MH, Bourdi M, Truog WE. Knockdown of ERp57 increases BiP/GRP78 induction and protects against hyperoxia and tunicamycin-induced apoptosis. Am J Physiol Lung Cell Mol Physiol. (2009) 297:L44-51. doi: 10.1152/ajplung.90626.2008

47. Schogler A, Caliaro O, Brugger M, Oliveira Esteves BI, Nita I, Gazdhar A, et al. Modulation of the unfolded protein response pathway as an antiviral approach in airway epithelial cells. Antiviral Res. (2019) 162:44-50. doi: 10.1016/j.antiviral.2018.12.007

48. Fritz JM, Dong M, Apsley KS, Martin EP, Na CL, Sitaraman S, et al. Deficiency of the BiP cochaperone ERdj4 causes constitutive endoplasmic reticulum stress and metabolic defects. Mol Biol Cell. (2014) 25:431-40. doi: $10.1091 / \mathrm{mbc} . e 13-06-0319$ 
49. Barabutis N. Growth hormone releasing hormone in the unfolded protein response context. Endocrine.. (2020) 67:291-3. doi: 10.1007/s12020-020-02205-8

50. Uddin MA, Akhter MS, Singh SS, Kubra KT, Schally AV, Jois S, et al. GHRH antagonists support lung endothelial barrier function. Tissue Barriers. (2019) 7:1669989. doi: 10.1080/21688370.2019.1669989

51. Zhang C, Cai R, Lazerson A, Delcroix G, Wangpaichitr M, Mirsaeidi M, et al. Growth hormone-releasing hormone receptor antagonist modulates lung inflammation and fibrosis due to bleomycin. Lung. (2019) 197:541-9. doi: 10.1007/s00408-019-00257-w

52. Barabutis N, Dimitropoulou C, Birmpas C, Joshi A, Thangjam G, Catravas JD. p53 protects against LPS-induced lung endothelial barrier dysfunction. Am J Physiol Lung Cell Mol Physiol. (2015) 308:L776-87. doi: 10.1152/ajplung.00334.2014

53. Barabutis N, Dimitropoulou C, Gregory B, Catravas JD. Wildtype p53 enhances endothelial barrier function by mediating RAC1 signalling and RhoA inhibition. J Cell Mol Med. (2018) 22:1792-804. doi: $10.1111 /$ jcmm.13460

54. Akhter MS, Uddin MA, Barabutis N. Unfolded protein response regulates P53 expression in the pulmonary endothelium. J Biochem Mol Toxicol. (2019) 33:e22380. doi: $10.1002 /$ jbt. 22380

55. Barabutis N. Unfolded protein response in acute respiratory distress syndrome. Lung. (2019) 197:827-8. doi: 10.1007/s00408-019-00279-4

56. Kubra KT, Uddin MA, Akhter MS, Barabutis N. Hsp90 inhibitors induce the unfolded protein response in bovine and mice lung cells. Cell Signal. (2020) 67:109500. doi: 10.1016/j.cellsig.2019.109500

57. Chen F, Wang W, El-Deiry WS. Current strategies to target p53 in cancer. Biochem Pharmacol. (2010) 80:724-30. doi: 10.1016/j.bcp.2010.04.031

58. Barabutis N, Schally AV, Siejka A. P53, GHRH, inflammation and cancer. EBioMedicine. (2018) 37:557-62. doi: 10.1016/j.ebiom.2018.10.034

59. Uddin MA, Akhter MS, Siejka A, Catravas JD, Barabutis N. P53 supports endothelial barrier function via APE1/Ref1 suppression. Immunobiology. (2019) 224:532-8. doi: 10.1016/j.imbio.2019.04.008
60. Yang WJ, He JX, Zhou MX, Huang M, Wang SQ, Wang XN, et al. An isopentenyl-substituted flavonoid norartocarpin activates Nrf2 signalling pathway and prevents oxidative insults in human lung epithelial cells. Free Radic Res. (2019) 53:348-58. doi: 10.1080/10715762.2019.1582769

61. Esposito V, Grosjean F, Tan J, Huang L, Zhu L, Chen J, et al. CHOP deficiency results in elevated lipopolysaccharide-induced inflammation and kidney injury. Am J Physiol Renal Physiol. (2013) 304:F440-50. doi: 10.1152/ajprenal.00487.2011

62. Yung HW, Colleoni F, Dommett E, Cindrova-Davies T, Kingdom J, Murray AJ, et al. Noncanonical mitochondrial unfolded protein response impairs placental oxidative phosphorylation in early-onset preeclampsia. Proc Natl Acad Sci USA. (2019) 116:18109-18. doi: 10.1073/pnas.1907548116

63. Barabutis N. P53 in RhoA Regulation. Cytoskeleton. (2020) 77:197-201. doi: $10.1002 / \mathrm{cm} .21604$

64. Barabutis N, Schally AV. Growth hormone-releasing hormone: extrapituitary effects in physiology and pathology. Cell Cycle. (2010) 9:4110-6. doi: $10.4161 /$ cc.9.20.13787

65. Kubra KT, Akhter MS, Uddin MA, Barabutis N. P53 versus inflammation: an update. Cell Cycle. (2020) 19:160-2. doi: 10.1080/15384101.2019.1708575

66. Kubra KT, Akhter MS, Uddin MA, Barabutis N. Unfolded protein response in cardiovascular disease. Cell Signal. (2020) 73:109699. doi: 10.1016/j.cellsig.2020.109699

Conflict of Interest: The author declares that the research was conducted in the absence of any commercial or financial relationships that could be construed as a potential conflict of interest.

Copyright (C) 2020 Barabutis. This is an open-access article distributed under the terms of the Creative Commons Attribution License (CC BY). The use, distribution or reproduction in other forums is permitted, provided the original author(s) and the copyright owner(s) are credited and that the original publication in this journal is cited, in accordance with accepted academic practice. No use, distribution or reproduction is permitted which does not comply with these terms. 